\title{
Tod dem Arztkittel?!
}

\author{
Simone Scheithauer
}

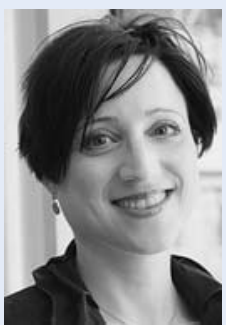

Simone Scheithauer

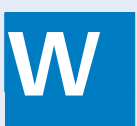

ieder hat ein krankenhaushygienisches Thema es geschafft, auf breites mediales Interesse zu stoßen. Diesmal jedoch kein Skandal, vielmehr die Lösung vieler Probleme: die Abschaffung eines Statussymbols, des Arztkittels! Diese - so wird es suggeriert - scheinbar längst überfällige Maßnahme werde es schaffen, multiresistente Erreger gleichsam wie nosokomiale Infektionen zu vermeiden.

Zahlreiche Anfragen zu Interviews folgen, gegebene Interviews werden nicht oder nur selten abgedruckt. Ganz offensichtlich waren die von einigen Kollegen getätigten Aussagen - sagen wir einmal - nicht ins Bild passend.

Was ist dies nun für eine neue Mode? Und warum ist sie es wert, sich näher mit ihr zu beschäftigen? Nun, wäre es nur eine Mode über die man unterschiedlicher Ansicht sein kann (wie das bei Moden nicht ganz ungewöhnlich ist), wäre es im Wesentlichen nicht der Rede wert.

Wäre es eine sinnvolle Maßnahme der Infektionsprävention, so wären wir dringend aufgerufen diese umzusetzen.

Wäre es eine Maßnahme, welche nicht nur nicht evidenz-basiert ist, sondern möglicherweise positive Aspekte einer offiziellen Bekleidung nicht genügend berücksichtigt, wären wir gefordert, uns mit ihr auseinander zu setzen.

Was steckt also dahinter?

Es gibt die schöne Geschichte, der weiße Arztkittel gehe auf den Schweizer Kaufmann und späteren Rotkreuz(Mit-)Begründer Henry Dunant zurück, der auf einer Reise zu Napoleon III. unvermittelt in die Schlacht von Solferino geriet und ohne Mandat und Auftrag den Verletzten und Angehörigen zu Hilfe kam. Er soll dies in seiner geschäftstypischen Bekleidung getan haben, also im weißen Hemd nach Ablegen des Sakkos. Weitere seiner auf die Reise mitgenommenen Hemden benutzte er als Verbandsmaterial. Diese geschichtliche Begebenheit ist auch von großer Bedeutung, da in ihr der
Grundstein der fünf Jahre später durch zwölf Nationen unterzeichneten 1.Genfer Konvention liegt.

Diese Anekdote zur potenziellen Herkunft des weißen Kittels ist eine Randnotiz für die aktuelle Frage; sie verdeutlicht jedoch ein ganz wesentliches Missverständnis auf das später eingegangen wird.

Um den Stellenwert des weißen Arztkittels rational beurteilen zu können, müssen wir uns folgende Fragen stellen:

1. Gibt es wissenschaftliche Evidenz, dass der weiße Arztkittel relevant ist für die Ätiologie nosokomialer Infektionen und insbesondere für die Übertragung multiresistenter Erreger?

2. „Was“ stellt der weiße Arztkittel dar (Gretchenfrage bezugnehmend auf die historische Episode in Solferino)?

3. Hat der weiße Arztkittel über seine mutmaßlich infektionsübertragende Bedeutung hinaus positive Eigenschaften, die wir im Sinne einer Güterabwägung berücksichtigen müssen?

Wie steht es also um die Evidenz des Arztkittels als Risikofaktor für Erregerübertragung und nosokomiale Infektionen?

Zunächst ein Blick über den deutschen Tellerrand. Es wird gerne darauf verwiesen, dass andere Nationen wie die Briten (und die Niederländer) diese fortschrittliche Maßnahme bereits lange eingeführt hätten. Das stimmt. 2007/2008 wurde per gesundheitsministeriellen Beschluss in Großbritannien ein generelles Verbot zum Tragen langärmeliger weißer Arztkittel ausgesprochen. Dieses Verbot stand jedoch nicht allein, sondern war Bestandteil der sogenannten „Bare below elbows“-Politik. Und hier kommt es zu einem Missverständnis: Gerne wird in der aktuellen Berichterstattung die Abschaffung der langärmeligen weißen Arztkittel mit der „Bare below elbows“-Strategie gleichgesetzt. Das ist jedoch so nicht zutreffend. Die Strategie umfasst viele unter anderem auch unfallschutz- und arbeitsschutz-rechtliche Elemente, wie die Vermeidung von Schmuck an Fingern und Unterarmen, das Tragen kur- 
zer, sauberer und nicht künstlicher Fingernägel, sowie eine adäquate Händehygiene. Der Arztkittel ist quasi „on top“ in dieses Bündel mit aufgenommen worden. Nach der Einführung dieser Direktive erhob sich in Großbritannien eine Welle großen Unmutes; insbesondere klinisch tätige Kollegen wiesen zu Recht auf die fehlende Evidenz für eben diese Maßnahme hin. In der Folge wurde in der britischen Empfehlung 2010 die Stringenz dieser Maßnahme zumindest gelockert [1-4].

Was wissen wir über den weißen Kittel als Vehikel der Infektionsübertragung?

Wir wissen, dass insbesondere die Ärmel und die Taschen weißer Arztkittel mit Bakterien kontaminiert sind und diese dort auch eine Zeit lang überleben können $[5,6]$. Wir wissen weiterhin, dass unter diesen Bakterien auch relevante nosokomiale Pathogene wie multiresistente Erreger sein können [7]. Es ist also theoretisch vorstellbar, dass weiße Kittel im Rahmen der Infektionsübertragung eine Vehikelfunktion haben könnten. Keine Studie konnte jedoch bislang belastbar zeigen, dass das Tragen weißer Kittel oder deren bakterielle Kontamination in der Praxis mit einer Übertragung von Erregern auf Patienten und/oder der Entstehung von nosokomialen Infektionen assoziiert ist $[1,2]$. Wir wissen auch, dass die bakterielle Erregerlast auf langärmeligen weißen Arztkitteln derjenigen gleicht, die kurzärmelige Kasacks nach acht Stunden Dienstzeit aufweisen [8].

Was heißt das?

Nun, es heißt das, was wir schon immer wussten: Bakterien sind überall, auch in uns, an uns und an unserer Kleidung. Wichtig ist, dass diese Bakterien, insbesondere die mit hoher Virulenz oder Resistenz, nicht zwischen den Patienten übertragen werden und insbesondere nicht an infektionsrelevante Stellen gelangen, z. B. an die Konnektionsstellen eines Infusionssystems, in eine Operationswunde.

Das führt unmittelbar zu Frage 2: Worüber reden wir, wenn wir über einen weißen Arztkittel reden?

Die mediale Darstellung suggeriert für den „medizinischen Laien“, dass die Maßnahme „Tragen langärmeliger Arztkittel“ quasi universell verbreitet ist und jetzt ersetzt wird durch die neue Maßnahme, das Tragen kurzärmeliger Alternativen. Dies trifft jedoch so nicht zu. Die differenzierend relevanten Begriffe sind hier Dienstkleidung versus Schutzkleidung, sowie infektionsrelevante Tätigkeiten und Risikobereiche [9]. Als Dienstkleidung gilt diejenige Kleidung die im betrieblichen Interesse anstelle der individuellen Zivilkleidung zur besonderen Kenntlichmachung zu tragen ist (BRG
19. Mai 1989 - 9 AZR 307 (96-BAGE 89, 26,30). Bezogen auf unsere Situation: der Patient erkennt den Arzt an seiner Dienstkleidung, nämlich am weißen Kittel. Streng davon abzutrennen ist jedoch die sogenannte Schutzkleidung, welche in allen Situationen mit erhöhten Anforderungen an die Infektionsprävention angelegt wird, z.B. sterile OP-Kittel des OP-Teams, unsterile Einmalschutzkittel bei der körperlichen Untersuchung eines MRSA-positiven Patienten, etc. Der Arztkittel zählt nicht zur Schutzkleidung und darf in solchen Situationen nicht getragen werden. Darüber hinaus wird in zahlreichen Bereichen des Krankenhauses bei der direkten Arbeit am Patienten der Arztkittel routinemäßig abgelegt (Achtung: anders als seinerzeit in Solferino und anders als in der aktuellen medialen Darstellung suggeriert!). Abgelegt wird er z. B., wenn er der korrekten hygienischen Händedesinfektion im Wege steht, wie bei einem Wundverbandswechsel, aber auch generell im Rahmen der Patientenversorgung in Risikobereichen wie Intensivstationen. Das heißt, ein wesentliches Element der Fehlwahrnehmung ist die nicht präzise Trennung der Begriffe Schutzkleidung und Dienstkleidung sowie der tatsächliche Ablauf vieler Prozesse im Krankenhausalltag.

Die Antworten auf die Fragen 1 und 2 zeigen, dass das mögliche Problem nicht a priori im Arztkittel selbst liegt, sondern in fehlerbehafteten Prozessabläufen. Diese Aspekte sind jedoch hinlänglich bekannt und müssen durch Schulungen kommuniziert und vorgelebt werden.

Das heißt, in der Konsequenz kann die Klinikleitung das Tragen langärmeliger weißer Arztkittel natürlich untersagen. Nichtsdestotrotz müssen wir auch dann die Ärzte dazu motivieren, ihre nun kurzärmeligen Alternativen regelmäßig zu wechseln und diese nicht mit einem Schutzkittel zu verwechseln. Unsere klinischen Kollegen sind so kompetent, selber zu entscheiden, wann sie welche Kleidung auswählen. Es mag Argumente für die Abschaffung der langärmeligen weißen Arztkittel geben, auch den unglaublich geschickten Werbefaktor.

Aber wir haben noch nicht das ganze Bild betrachtet, kommen wir also zu Punkt 3: Hat der weiße Arztkittel irgendeinen Zusatznutzen jenseits krankenhaushygienischer Fragestellungen:

Ja den scheint es zu geben, zwei Aspekte sind von Relevanz: Die meisten Untersuchungen zu dieser Frage haben gezeigt, dass die Mehrheit aller Patienten Ärzte mit weißem Arztkittel bevorzugen [10]. Einige Arbeiten untersuchten explizit die Akzeptanz des Tragens kurzärmeliger Arztkleidung, diese wurde in den meisten 
Fällen als am wenigsten geeignet empfunden. Patienten assoziieren mit dem Tragen langärmeliger Arztkittel im Vergleich zu der kurzärmeligen Variante Vertrauen und Ermutigung durch den behandelnden Arzt Dabei wurde ihre Information nicht durch das Wissen um potenzielle Risiken via Arztkittel übertragender Erreger respektive Infektionen beeinflusst. Dies ist umso bedeutsamer, als dass letztgenannter Punkt eine große Furcht der meisten Patienten im Krankenhaus darstellt [11 - 13]. Auf der anderen Seite gibt es einige Untersuchungen insbesondere aus Australien oder aus dem Bereich der Allgemein- und Familienmedizin, in der die Patienten keine klare Präferenz für eine Bekleidungsform angaben. Weiterhin interessant ist, dass Patienten anscheinend Mitarbeitern im Gesundheitssystem mehr Vertrauen entgegenbringen, wenn diese offizieller gekleidet sind, auch wenn sie diesen Stil nicht persönlich favorisieren [10,13,14]. Diese Daten sind von hoher Relevanz, insbesondere wenn man sich auch nur die Grundzüge des Potenzials des Placeboeffektes und darüber hinaus die Rolle sogenannter weicher Faktoren bei der Arzt-Patienten-Bindung und der Behandlung chronischer Erkrankungen vor Augen führt. Nicht unerwähnt bleiben soll auch der negative Einfluss des weißen Arztkittels im Sinne der sogenannten „white coat hypertension“ [15].

Möglicherweise handelt es sich bei allen aufgezeigten Phänomenen nicht um statische Aussagen, sondern um eine dynamische Wahrnehmung, die sich - durch neue Standards - über Generationen verändern kann.

Kleider machen also Leute, aber gilt das nur für das Gegenüber?

Nein, wie eine Studie von Psychologen aus dem USBundesstaat Illinois aufzeigen konnte [16]. Sie ließen Testpersonen Aufgaben lösen, um deren Konzentration und Reaktionsvermögen zu untersuchen; die eine Hälfte trug Alltagskleidung, die andere trug weiße langärmelige Arztkittel. Das Ergebnis war frappierend. Die Teilnehmer im weißen Arztkittel zeigten signifikant bessere Studienergebnisse. Auch eine Modifikation des Experimentes kam zu bemerkenswerten Ergebnissen: Nun trugen alle Probanden einen weißen Kittel, welcher der einen Hälfte als Arztkittel, der anderen Hälfte als Anstreicherkittel benannt wurde. Das Konzentrationsvermögen der ersten Gruppe war deutlich größer als das der zweiten Gruppe. Die Arbeitsgruppe bezeichnet das hier aufgedeckte Phänomen als „enclothed cognition“ (bekleidete Wahrnehmung) und bezeichnet damit die gleichzeitige Wirkung von zwei Faktoren: Die symbolische Bedeutung eines Kleidungsstückes und die körperliche Erfahrung es zu tragen.
Was bleibt festzuhalten?

- Die Abschaffung des weißen Arztkittels ist eine Maßnahme, deren infektionspräventiver Effekt bisher nicht belegt ist; auf jeden Fall stellt sie ein sehr effektives Marketinginstrument dar.

- Auch ohne Abschaffung des weißen Arztkittels kann eine adäquate Infektionsprävention betrieben werden; entscheidend sind die Arbeitsabläufe und der korrekte Einsatz.

- Die Fokussierung auf eine nicht-evidenz-basierte Maßnahme als „Schlüssel zum Erfolg“ bietet zwei Gefahren: Einerseits täuscht sie vor, was die Öffentlichkeit sowieso glaubt: Infektionsvermeidung ist einfach und die Herausforderung Multiresistenz ist mit trivialen Methoden zu lösen. Wir alle wissen, dass dies ein gefährlicher Trugschluss ist. Zum zweiten könnte durch falsche Fokussierung auf eine einzelne nicht-evidenz-basierte Maßnahme die Umsetzung vieler evidenz-basierter Maßnahmen in den Hintergrund geraten. Dies hätte eine reale Infektionsgefahr für den Patienten zur Folge und darf nicht passieren.

- Der weiße Arztkittel hat jenseits aller Aspekte der Krankenhaushygiene ganz offensichtlich noch andere nicht zu vernachlässigende Funktionen.

Aus meiner Sicht folgt daraus, dass es jedem selber überlassen sein sollte, ob er den langärmeligen Arztkittel weiterhin einsetzen möchte - an den Stellen und in den Situationen in denen es adäquat und sinnvoll ist. Diese Entscheidung kann auch gemeinsam für einzelne Stationen oder Institutionen getroffen werden. Für diejenigen, die die „neue Mode“ mitmachen möchten, gilt aber: Vorsicht, dass nicht eine nur symbolische und fraglich effektive Maßnahme in den Vordergrund rückt, infektionspräventive Maßnahmen mit nachgewiesener Effektivität aber vernachlässigt werden. Darüber hinaus sollten potenzielle unerwünschte Nebenwirkungen beachtet werden.

Fazit: Seien Sie modisch oder aber nicht - verlieren Sie jedoch nicht Ihren Stil.

Denn - um es mit Coco Chanel zu sagen: „Mode ist vergänglich. Stil niemals“. 


\section{Referenzen}

1 Henderson J. The endangered white coat. Clin Infect Dis 2010; 50: $1073-1074$

2 Pratt RJ, Pellowe CM, Wilson JA et al. epic2: National evidencebased guidelines for preventing healthcare-associated infections in NHS hospitals in England. J Hosp Infect 2007; 65 : 011 S64

3 Department of Health UK. Uniforms and workwear: an evidence base for developing local policy (17.09.2007). Im Internet: http://webarchive.nationalarchives.gov.uk/ 20130107105354/http://www.dh.gov.uk/en/Publicationsandstatistics/Publications/PublicationsPolicyAndGuidance/ DH_078433 Stand: 11.5.2016

4 Clement R. Is it time for an evidence based uniform for doctors? BMJ 2012; 345: e8286DOI DOI: 10.1136/bmj.e8286

5 Wong D, Nye K, Hollis P. Microbial flora on doctors' white coats. BM] 1991; 303: $1602-1604$

6 Loh W, Ng VV, Holton J. Bacterial flora on the white coats of medical students. J Hosp Infect 2000; 45: 65-68

7 Treakle AM, Thom KA, Furuno JP et al. Bacterial contamination of health care workers' white coats. Am J Infect Control 2009; 37: $101-105$

8 Burden M, Cervantes L, Weed D et al. Newly cleaned physician uniforms and infrequently washed white coats have similar rates of bacterial contamination after an 8-hour workday: a randomized controlled trial. J Hosp Med 2011; 6: 177-182

9 Rüden H, Schulze-Röbbecke R. Kleidung des medizinischen Personals - Bedeutung und Einsatz aus krankenhaushygienischer Sicht. Krankenhaushygiene up2date 2007; 2: 97 - 112

10 Landry M, Dornelles AC, Hayek G et al. Patient Preferences for Doctor Attire: The White Coat's Place in the Medical Profession. Ochsner J 2013; 13: 334-342

11 Douse J, Derrett-Smith E, Dheda K et al. Should doctors wear white coats? Postgrad Med J 2004; 80: 284-286

12 Rehman SU, Nietert PJ, Cope DW et al. What to wear today? Effect of doctor's attire on the trust and confidence of patients Am J Med 2005; 118: 1279-1286

13 Gherardi G, Cameron J, West A et al. Are we dressed to impress? A descriptive survey assessing patients' preference of doctors' attire in the hospital setting Clin Med (Lond) 2009; 9: $519-524$

14 Toquero L, Aboumarzouk O, Owers C et al. Bare Below The Elbows- The Patient's Perspective. WebmedCentral QUALITY AND PATIENT SAFETY 2011; 2: WMC001401 DOI 10.9754/ journal.wmc.2011.001401

15 Angeli F, Verdecchia P, Gattobigio R et al. White-coat hypertension in adults. Blood Press Monit 2005; 10: 301 - 305

16 Adam H, Galinsky AD. Enclothed cognition. J Experiment Soc Psych 2012; 48: $918-925$ 